\title{
Temporary synchronous ventricular pacing as a bridge therapy after CRT-D removal due to lead-related infective endocarditis
}

\author{
Leszek Blicharz ${ }^{1, \mathrm{C}-\mathrm{D}, \mathrm{F}}$, Marcin Michalak ${ }^{1, \mathrm{~B}-\mathrm{C}, \mathrm{F}}$, Andrzej Cacko ${ }^{1, \mathrm{~A}, \mathrm{E}-\mathrm{F}}$, Robert Kowalik ${ }^{1, \mathrm{~B}, \mathrm{~F}}$, \\ Marcin Grabowski ${ }^{1, A-F}$ \\ A - Research concept and design, B - Collection and/or assembly of data, C - Data analysis and interpretation, \\ D - Writing the article, E - Critical revision of the article, F - Final approval of article
}

1 1st Department of Cardiology, Medical University of Warsaw

Address for correspondence:

Leszek Blicharz, 1st Department of Cardiology, Medical University of Warsaw email: 1blicharz@gmail.com Marcin Michalak, 1st Department of Cardiology, Medical University of Warsaw
email: marcin.michalak@wum.edu.pl

Andrzej Cacko, 1st Department of Cardiology, Medical University of Warsaw
email: andrzej.cacko@wum.edu.pl

Robert Kowalik, 1st Department of Cardiology, Medical University of Warsaw email: rjkowalik61@gmail.com

Marcin Grabowski, 1st Department of Cardiology, Medical University of Warsaw email: marcin.grabowski@wum.edu.pl

Received: 15.12.2016

Revised: 28.12.2016

Accepted: 28.12.2016

\section{Key words:}

cardiac resynchronization therapy, lead-related infective endocarditis, temporary pacing

We present a case of single-lead temporary synchronous pacing in a patient after cardiovascular implantable electronic device (CIED) removal.

A 60-year-old stimulation-dependent patient implanted with cardiac resynchronization therapy and defibrillator (CRT-D) was admitted to the hospital due to heart failure decompensation. Past medical history included chronic heart failure with low ejection fraction (EF), dilated cardiomyopathy, episodes of ventricular arrhythmia, paroxysmal atrial fibrillation (AF) and decompensated mitral regurgitation. The patient developed lead-related infective endocarditis which necessitated complete removal of the implanted system. A spontaneous sinus rhythm (rate 80-100 BPM) was present, but due to complete heart block and ventricular dyssynchrony significant heart failure (HF) decompensation would have occurred without an intervention. Temporary ventricular stimulation was introduced as a bridge therapy until the eradication of the infective endocarditis. Taking advantage of the sinus rhythm, the patient was provided with a Medtronic SEDR01 Sensia DR pacemaker set to VDD mode and connected to the Biotronik Linox Smart ProMRI S DX 65/15, which resulted in single-lead atrial-synchronized ventricular pacing. The lead was introduced through the left subclavian vein into the right ventricular apex. The pacemaker and the proximal part of the lead were externalized outside of the infected pocket in the left subclavian area (Figure 1). Peri-procedure lead parameters

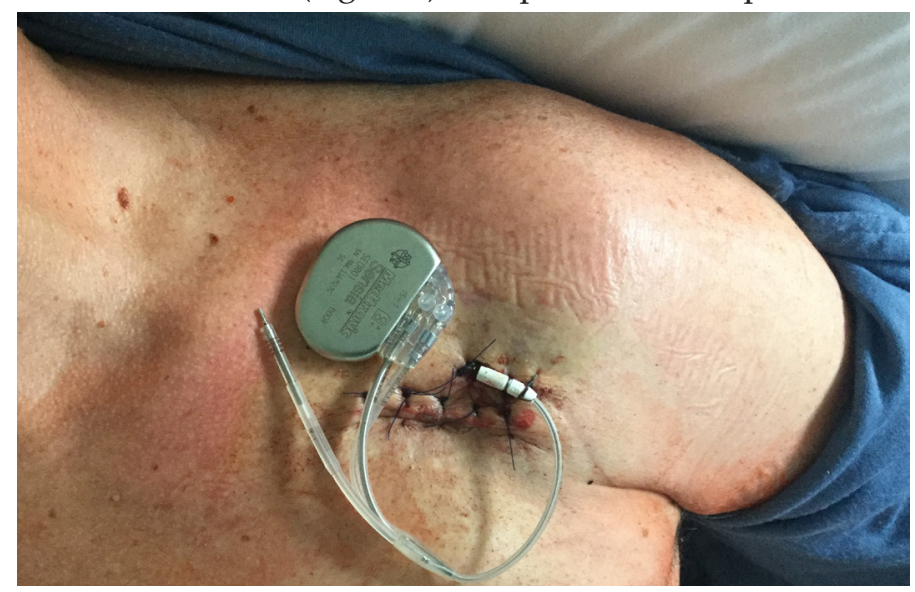

Figure 1 Medtronic SEDR01 Sensia DR as a temporary pacemaker connected to Biotronik Linox Smart ProMRI S DX 65/15 lead tunneled in the skin and emerging outside of the infected pocket. 
were acceptable. Device control showed that the measured $\mathrm{P}$ wave amplitude of $0.35-0.5 \mathrm{mV}$ was sufficient for adequate atrial sensing ( $\mathrm{AsVp}=99.9 \%$ ) (Figure 2, Figure 3). During the follow-up there were no recorded episodes of supraventricu-

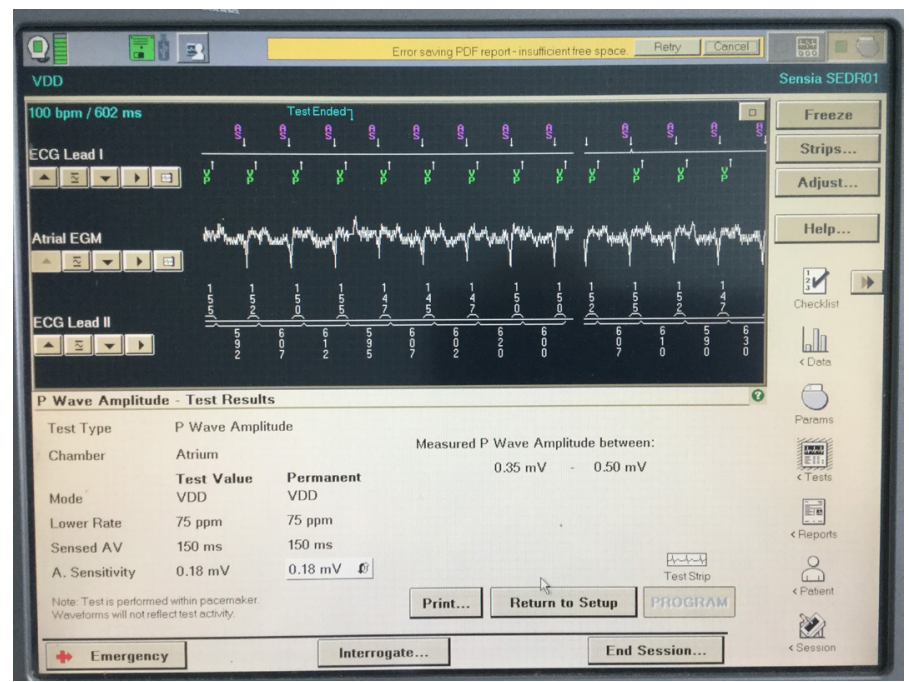

Figure 2 Measured $\mathrm{P}$ wave amplitude range during pacemaker test: 0.35-0.5 mV. Atrial sensitivity programmed to $0.18 \mathrm{mV}$.

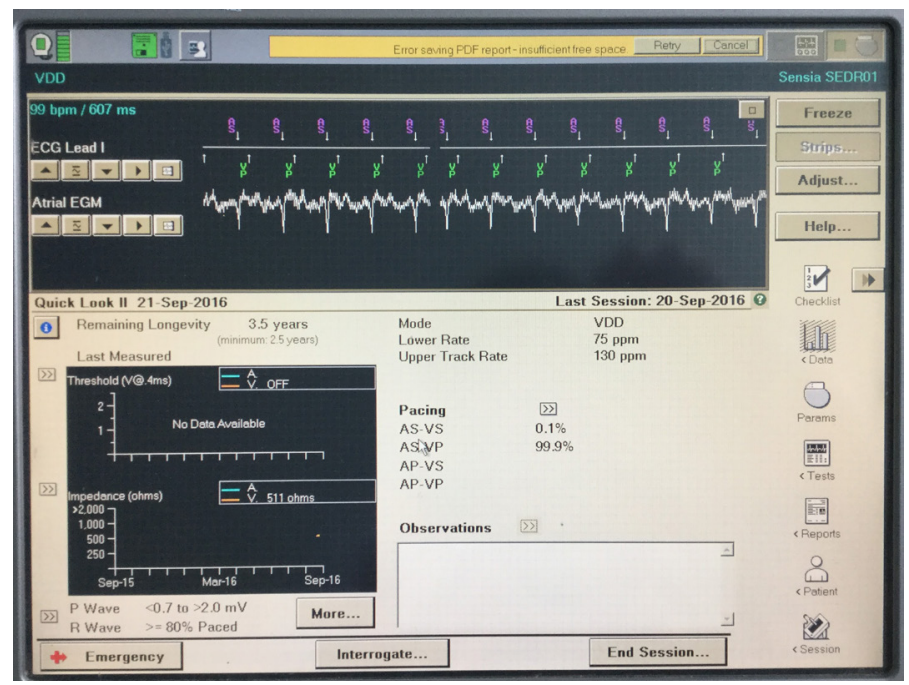

Figure 3 High percentage of atrial-synchronized ventricular pacing ( $A s \vee p=99.9 \%$ ) recorded during follow-up.

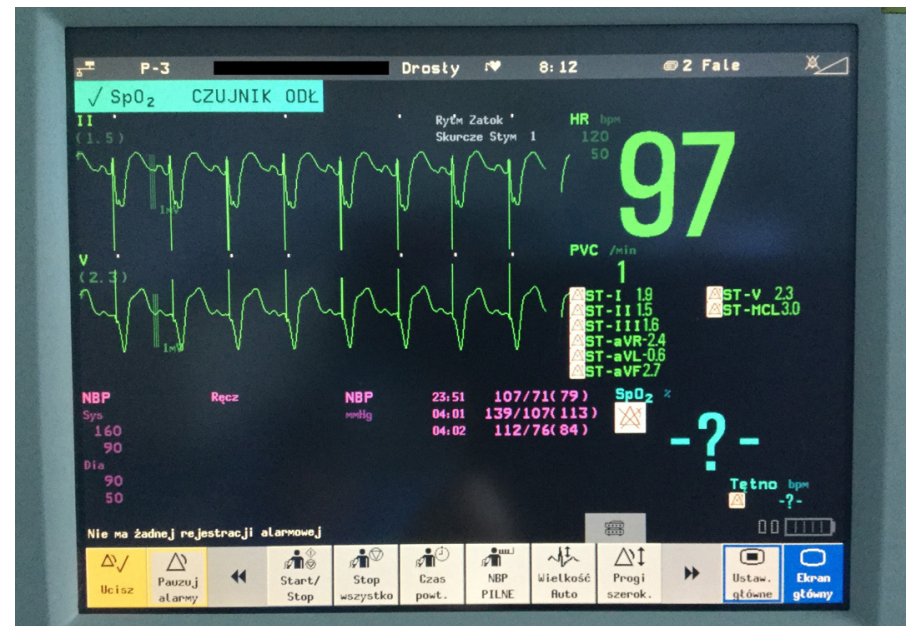

Figure 4 ECG monitoring consistent with device control findings. Atrioventricular synchrony visibly present. lar arrhythmia. Data from ECG monitoring also showed that sensing and stimulation were successful (Figure 4).

Infective endocarditis was initially treated empirically with gentamicin and vancomycin. Blood culture revealed Enterococcus faecalis bacteraemia. The isolated strain was sensitive to ampicillin, which was subsequently introduced instead of former antibiotics. The treatment was maintained for a total of 6 weeks. The patient is planned to be electively implanted with a new CRT-D contralaterally.

\section{Discussion}

Complete CIED removal is an obligatory procedure in case of lead-related infective endocarditis [1]. It may cause serious complications in stimulation-dependent patients, who require another method of pacing until the system can be replaced. A standard approach involves insertion of a temporary endocardial bipolar lead into the right ventricle and connecting it to an external pacemaker. It has been recently reported that active bipolar leads designed for permanent cardiac stimulation may also be used with a pacemaker in VVI mode [2]. Such a solution usually ensures satisfactory pacing support but does not provide atrioventricular synchrony in case of complete heart block. Hence, as long as this situation persists, patients with preserved sinus rhythm could experience pacemaker syndrome or decompensation of pre-existing heart failure. In our setting addition of an atrial lead would be cumbersome and carry unnecessary risk for the patient. With that in mind, we experimentally implemented another solution in order to maximally alleviate the HF symptoms.

Biotronik DX provides dual-chamber diagnostics in a single-lead ICD device. The Linox Smart ProMRI S DX 65/15 lead with floating sensing atrial dipole has proven to be a safe and efficient tool for discrimination between supraventricular and ventricular arrhythmias as well as for diagnostics of the atria. The idea behind this concept is to ensure safe antiarrhythmic therapy while limiting the amount of hardware, i.e. an additional atrial lead. The DX ICD family has a specific signal amplification and filtration circuitry designed for registration of atrial signals. It varies from circuitry found in typical dual chamber pacemakers with active or passive fixation of the atrial lead directly in the atrial wall. Nevertheless, our case proves that DX leads connected to a temporary VDD pacemaker can be used for synchronous ventricular pacing. During the implantation procedure it is possible to verify whether the pacing system analyzer records an adequate signal through the floating electrode.

Some additional benefits of using an ICD lead included decreased risk of dislocation, which is mainly attributed to its lower flexibility when compared to standard pacing leads. Thanks to the DX lead we could also possibly assess whether the patient had periods of supraventricular arrhythmia during the follow-up (none were recorded in this case).

CIED removal was necessitated by infection caused by Enterococcus faecalis. This microorganism is responsible for approximately $90 \%$ of cases of enterococcal infective endocarditis [3]. It may cause numerous therapeutic problems (possible antibiotic resistance, necessity of long-term treatment). 
Empiric choice of drugs was verified by blood culture and antibiogram. In order to ensure disease eradication, ampicillin administration was maintained for 6 weeks.

\section{Conclusions}

To the best of our knowledge, this report is the first example of single-lead temporary synchronous pacing in a patient after CIED removal. Due to predicted long-term antibiotic treatment in infective endocarditis and delayed CRT-D system replacement, we aimed to reach optimum heart failure compensation by means of providing AV synchrony. Success of DX lead implantation (adequate atrial sensing, AsVp $=99.9 \%$ ) may encourage new investigations with regards to patients with AV conduction disorders.

\section{References}

1. Transvenous lead extraction: Heart Rhythm Society expert consensus on facilities, training, indications, and patient management: this document was endorsed by the American Heart Association (AHA). Wilkoff BL, Love CJ, Byrd CL et al., Heart Rhythm. 2009 Jul;6(7):1085-104.

2. Long-term temporary pacing with an active fixation lead. Maciąg A, Syska P, Oręziak A et al., Kardiol Pol. 2015;73(12):1304-9.

3. 2015 ESC Guidelines for the management of infective endocarditis: The Task Force for the Management of Infective Endocarditis of the European Society of Cardiology (ESC) Endorsed by: European Association for Cardio-Thoracic Surgery (EACTS), the European Association of Nuclear Medicine (EANM). Habib G, Lancellotti P, Antunes MJ et al., Eur Heart J. 2015 Nov 21;36(44):3075-128. 